\title{
Search for Trapped Antihydrogen: First Candidate Events
}

R. Hydomako ${ }^{* a}$, G.B. Andresen ${ }^{b}$, M.D. Ashkezari ${ }^{c}$, M. Baquero-Ruiz $^{d}$, W. Bertsche ${ }^{e}$, P.D. Bowe ${ }^{b}$, C.C. Bray ${ }^{d}$, E. Butler ${ }^{e}$, C.L. Cesar $^{f}$, S. Chapman ${ }^{d}$, M. Charlton ${ }^{e}$, J. Fajans $^{d}$, T. Friesen ${ }^{a}$, M.C. Fujiwara ${ }^{g, a}$, D.R. Gill ${ }^{g}$, J.S. Hangst ${ }^{b}$, W.N. Hardy ${ }^{h}$, R.S. Hayano $^{i}$, M.E. Hayden ${ }^{c}$, A.J. Humphries ${ }^{e}$, S. Jonsell ${ }^{e, j}$, L. V Jørgensen ${ }^{e}$, L. Kurchaninov $^{g}$, R. Lambo ${ }^{f}$, N. Madsen ${ }^{e}$, S. Menary ${ }^{k}$, P. Nolan ${ }^{l}$, K. Olchanski ${ }^{g}$, A. Olin $^{g}$, A. Povilus ${ }^{d}$, P. Pusa ${ }^{l}$, F. Robicheaux ${ }^{m}$, E. Sarid ${ }^{n}$, S. Seif El Nasr-Storey ${ }^{h}$, D.M. Silveira $^{o, p}$, C. So ${ }^{d}$, J.W. Storey ${ }^{g}$, R.I. Thompson ${ }^{a}$, D.P. van der Werf $^{e}$, D. Wilding ${ }^{e}$, J.S. Wurtele ${ }^{d}$, Y. Yamazaki ${ }^{o, p}$

${ }^{a}$ Department of Physics and Astronomy, University of Calgary, Calgary AB, T2N 1N4, Canada

${ }^{b}$ Department of Physics and Astronomy, Aarhus University, DK-8000 Aarhus C, Denmark

${ }^{c}$ Department of Physics, Simon Fraser University, Burnaby BC, V5A 1S6, Canada

${ }^{d}$ Department of Physics, University of California, Berkeley, CA 94720-7300, USA

${ }^{e}$ Department of Physics, Swansea University, Swansea SA2 8PP, United Kingdom

${ }^{f}$ Instituto de Física, Universidade Federal do Rio de Janeiro, Rio de Janeiro 21941-972, Brazil

${ }^{g}$ TRIUMF, 4004 Wesbrook Mall, Vancouver BC, V6T 2A3, Canada

${ }^{h}$ Department of Physics and Astronomy, University of British Columbia, Vancouver BC, V6T 1Z4,

Canada

${ }^{i}$ Department of Physics, University of Tokyo, Tokyo 113-0033, Japan

${ }^{j}$ Fysikum, Stockholm University, SE-10609, Stockholm, Sweden

${ }^{k}$ Department of Physics and Astronomy, York University, Toronto, ON, M3J 1P3, Canada

${ }^{l}$ Department of Physics, University of Liverpool, Liverpool L69 7ZE, United Kingdom

${ }^{m}$ Department of Physics, Auburn University, Auburn, AL 36849-5311, USA

${ }^{n}$ Department of Physics, NRCN-Nuclear Research Center Negev, Beer Sheva, IL-84190, Israel

${ }^{o}$ Atomic Physics Laboratory, RIKEN, Saitama 351-0198, Japan

${ }^{p}$ Graduate School of Arts and Sciences, University of Tokyo, Tokyo 153-8902, Japan

\begin{abstract}
ALPHA (Antihydrogen Laser PHysics Apparatus) is an international project located at CERN whose prime goal is to perform tests of CPT symmetry on antihydrogen. While substantial numbers of antihydrogen atoms have been produced in several experiments, their trapping has not yet been achieved. In the ALPHA apparatus, cold plasmas of typically $3 \times 10^{4}$ antiprotons and of $4 \times 10^{6}$ positrons are mixed in a Penning trap to form cold antihydrogen. A multipolar magnetic trap of depth 0.5 Kelvin is superimposed on the Penning trap to confine the anti-atoms. In 2009, we have reached, for the first time, the detection sensitivity and trap conditions for which observation of antihydrogen trapping may be expected. In this talk, we will present results from that run, where we have observed first candidate events [1].
\end{abstract}

35th International Conference of High Energy Physics

July 22-28, 2010

Paris, France

${ }^{*}$ Speaker. 


\section{Introduction and Apparatus}

Antihydrogen, the bound state of an antiproton and a positron, is the simplest antiatomic system. Moreover, antihydrogen can be directly compared to hydrogen as a stringent $C P T$ test. The ALPHA (Antihydrogen Laser PHysics Apparatus) project, located at the Antiproton Decelerator at CERN, is an international collaboration with the goal of magnetically confining, and performing such measurements on antihydrogen. The first step towards precision measurements is the stable confinement of antihydrogen on timescales necessary for microwave or laser spectroscopy.

The ALPHA apparatus combines a Penning trap for charged particles with a magnetic octupole and two mirror coils to confine neutral antihydrogen (Fig. 1, left). The two species are brought together to form antihydrogen in the magnetic neutral atom trap. While the neutral trap is engaged, an electric field is applied to the trap volume to eject charged particles. The neutral trap can then be quickly ramped down to reveal anything which had remained magnetically trapped inside.



a)

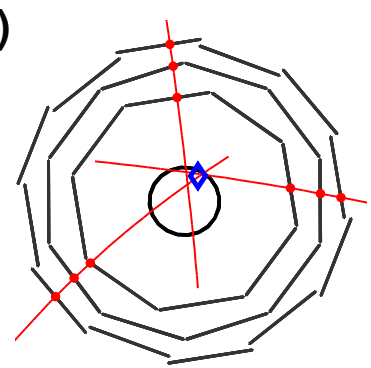

b)

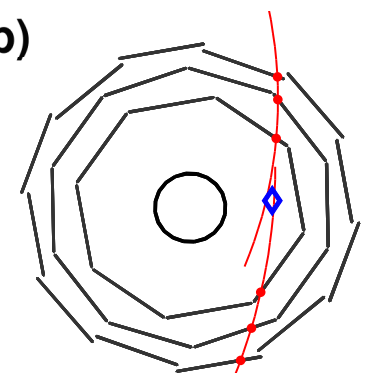

Figure 1: (Left) Cut-away of the ALPHA apparatus. Only components relevant to the trapping experiment are shown. (Right) Example reconstructions of an (a) antihydrogen annihilation, and (b) a cosmic ray.

\section{Event Reconstruction and Candidate Events}

To detect and study the antihydrogen stored or produced, ALPHA has surrounded the trapping volume with a tracking detector (Fig. 1, left). We are interested in the primary antiproton annihilation position (Fig. 1a). However, annihilations are not the only events to which our detector is sensitive. Cosmic ray events (Fig. 1b) are a significant source of background. To this end, cuts on the reconstruction parameters were carefully chosen by optimizing the expected p-value using calibration signal and background data. This resulted in a final cosmic pass rate of $2.2 \times 10^{-2} \mathrm{~Hz}$.

During the 2009 experimental run, 212 trapping experiments were performed, each with a detection window of $30 \mathrm{~ms}$. From these experiments, 6 candidate events, satisfying all of the cuts, were observed. This is compared to an expected number of background events of 0.14 . Thus, we find that the p-value for the rejection of the background hypothesis, that is, that the observed events are due to cosmic rays, is $9.2 \times 10^{-9}$, or $5.6 \sigma$.

It is important to note that, in addition to antihydrogen, bare antiprotons can also be confined within the neutral trap and result in an annihilation signal in the detector. Although a pulsed electric field is applied to clear away the charged particles, antiprotons with large transverse energies can remain mirror trapped in the neutral trap magnetic field.

To investigate the difference between trapped antihydrogen and mirror-trapped antiprotons, extensive simulations were performed. Fig. 2a shows the simulated distribution (in grey) of mirror- 
trapped antiprotons after the neutral trap ramp-down, while Fig. $2 \mathrm{~b}$ shows the distribution for simulated antihydrogen atoms. The 6 candidate events are shown in blue and appear to lie outside the regions identified for mirror-trapped antiprotons, but inside the expected region for neutral, trapped antihydrogen [1]. However, without adequate validation of the simulations and an unambiguous control experiment, we cannot definitively claim to have observed trapped antihydrogen ${ }^{1}$.
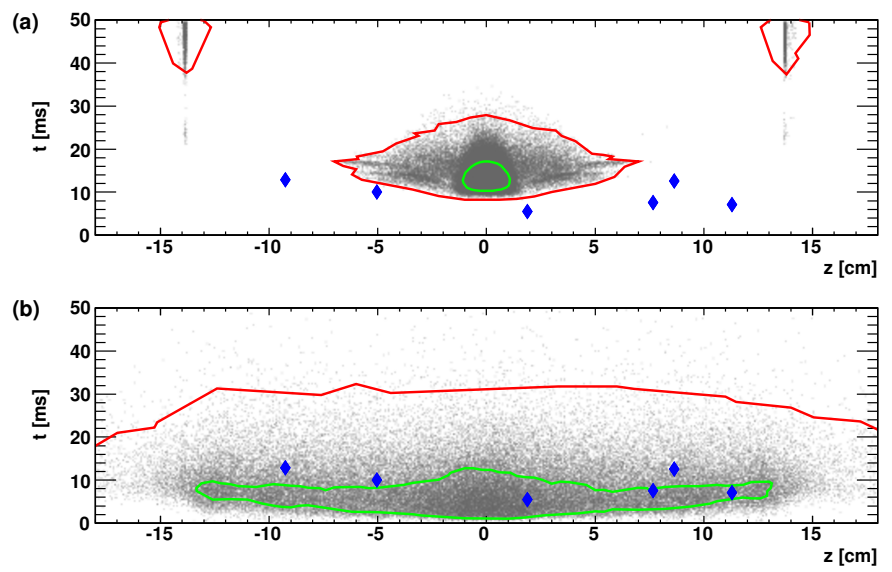

Figure 2: The time after the magnet shutdown and the z-position of the simulated annihilations of (a) mirrortrapped antiprotons and (b) antihydrogen atoms. The green contour contains $50 \%$ of the density of simulated events, while the red contours contain $99 \%$. The six observed candidate events are shown in blue.

\section{Future Measurements}

A possible spectroscopic scheme for trapped antihydrogen atoms involves using microwave radiation to induce a spin-flip in a trapped antihydrogen atom, resulting in a transition from a lowfield seeking state into a high-field seeking state. The high-field seeking atoms will be unconfined, and be detected as they reach the apparatus walls. These hyperfine transitions are well-known for hydrogen, and the comparison of the zero-field hyperfine splitting could be made in our apparatus (optimistically) to 1 part in $10^{6}$.

\section{Acknowledgments}

This work was supported by CNPq, FINEP/RENAFAE (Brazil), ISF (Israel), MEXT (Japan), FNU (Denmark), VR (Sweden), NSERC, NRC/TRIUMF, AIF (Canada), DOE, NSF (USA), EPSRC and the Leverhulme Trust (UK).

\section{References}

[1] G. B. Andresen et al. (ALPHA Collaboration), Search for Trapped Antihydrogen, Phys. Lett. B, DOI:10.1016/j.physletb.2010.11.004 (2010).

[2] G. B. Andresen et al. (ALPHA Collaboration), Trapped Antihydrogen, Nature, DOI:10.1038/nature09610 (2010).

\footnotetext{
${ }^{1}$ Note that after this conference, we reported the trapping of antihydrogen [2].
} 